\title{
2D ELECTRON GAS IN NON-UNIFORM MAGNETIC FIELDS
}

\author{
B.L. Gallagher ${ }^{a}$, V. Kubrak ${ }^{a}$, A.W. Rushforth ${ }^{a}$, A.C. Neumann ${ }^{a}$, \\ K.W. EDMONDS ${ }^{a}$, P.C. MAIN ${ }^{a}$, M. HenINI ${ }^{a}$, C.H. MARROWS ${ }^{b}$, \\ B.J. HickeY ${ }^{b}$, S. Thoms ${ }^{c}$ AND D.E. DAHLBERG ${ }^{d}$ \\ ${ }^{a}$ School of Physics and Astronomy, University of Nottingham \\ Nottingham NG7 2RD, U.K. \\ ${ }^{b}$ Department of Physics, University of Leeds, Leeds LS2 9JT, U.K. \\ ${ }^{c}$ Nanoelectronics Research Center, University of Glasgow, Glasgow G12 8QQ, U.K. \\ ${ }^{d}$ Department of Physics, University of Minnesota, MN 55455, USA
}

We present a review of some of our recent experimental work on hybrid ferromagnet/semiconductor devices in which $2 \mathrm{D}$ electrons propagate through magnetic barriers, periodic magnetic modulations, and random magnetic fields.

PACS numbers: 73.20.Dx, 73.50.Fq, 73.23.Ad, 73.50.Jt

\section{Introduction}

The theory of $2 \mathrm{D}$ electrons in non-uniform magnetic fields has an extensive research literature in which a range of new semiclassical and quantum effects are predicted [1-3]. Until recently there have been very few experimental studies however. A two-dimensional electron gas (2DEG) subject to a magnetic field which varies spatially on a length scale which is longer than the electron mean free path can be considered to have a spatially varying conductivity tensor [4]. This can lead, for example, to large magnetoresistances due the admixture of longitudinal and Hall resistances [4]. The more interesting case, which we focus on in this paper, is that of large amplitude magnetic fields that vary on length scales that are small compared to the electron mean free path. In this case the Lorentz force can strongly modify the electron trajectories leading to a wide range of new semiclassical effects. The nature of the electron states of the 2DEG will also be dependent on the form of the magnetic field leading to the possibility of new quantum mechanical effects.

In this paper we will be concerned with the situation in which the Zeeman energies are negligibly small. It is then only the component of the magnetic field perpendicular to the 2DEG that affects the electron states. One approach to the realisation of large amplitude spatially varying magnetic fields is the use 
of non-planar 2DEGs produced by re-growth on lithographically patterned substrates [5, 6]. Application of a uniform external magnetic field to a non-planar 2DEG then results in a spatially varying component of the magnetic field perpendicular to the 2DEG. So far devices produced in this way have been in the diffusive limit in which the magnetic field varies on length scales long compared to the mean free path.

The more direct approach, which several groups [7-23] have pursued, is the fabrication of lithographically patterned ferromagnetic structures above near-surface 2DEGs. Micron or submicron ferromagnetic elements such as magnetic dots and stripes can be used to produce a range of field profiles at the 2DEG. Such devices can be used to study the fundamental physics of electrons in non-uniform magnetic fields. Such hybrid devices also have a number of potential applications $[1,16,22,23]$ : (i) magnetic field sensors in which the ferromagnetic element can be considered as an amplifier of magnetic flux; (ii) memory or logic elements in which the non-volatile magnetization state of the ferromagnetic "gate" determines the resistance state of the conducting channel; and (iii) nanomagnetometers in which the size of the semiconductor channel can be matched to that of the ferromagnetic structure of interest.

\section{Hybrid ferromagnetic/semiconductor devices}

We will discuss the results obtained for a number of devices with differing geometries. All are based on similar near-surface heterostructures. Typically, at helium temperatures, they have electron densities of 3 to $5 \times 10^{15} \mathrm{~m}^{-2}$, mobilities of 60 to $80 \mathrm{~m}^{2} \mathrm{~V}^{-1} \mathrm{~s}^{-1}$, and electron mean free paths of 6 to $8 \mu \mathrm{m}$. The 2DEG is formed at a (AlGa)As/GaAs heterointerface $35 \mathrm{~nm}$ to $50 \mathrm{~nm}$ beneath the surface of the heterostructure. When ferromagnetic stripes and gratings are used they are orientated normal to the [100] GaAs crystal direction, which is non-piezoelectric, to minimise any strain-induced electric modulation at the 2DEG due to the differential thermal contraction of the ferromagnet and the GaAs [24]. We will consider the 2DEG to lie in the $x-y$ plane and the current to flow in the $x$-direction. The component of the magnetic field perpendicular to the plane of the 2DEG is then the $z$-component, $B_{z}$.

\section{Large amplitude submicron simple magnetic barriers}

A simple large,amplitude magnetic barrier at the 2DEG can be produced using the geometry shown in Fig. 1a $[17,19,22,23]$. The results that we will present are for samples with Co films that are 300 to $600 \mathrm{~nm}$ thick, of length and width 100 to $200 \mu \mathrm{m}$, fabricated on top of a Hall bar which is 5 to $20 \mu \mathrm{m}$ wide. In the absence of an external magnetic field the magnetization, $M$, of the ferromagnetic element will lie in the $x-y$ plane due to the strong shape anisotropy. Application of an external magnetic field along the $x$-axis will orient $M$ along this direction. At the plane of the 2DEG $B_{z}$ is independent of $y$ and has a large peak as a function of $x . B_{z}$ has maximum amplitude beneath the edge of the element, $x=0$, and decays rapidly on a length scale of a few hundred nanometers (see Fig. 1a). The height of the peak is proportional to the $x$-component of $M$. By 


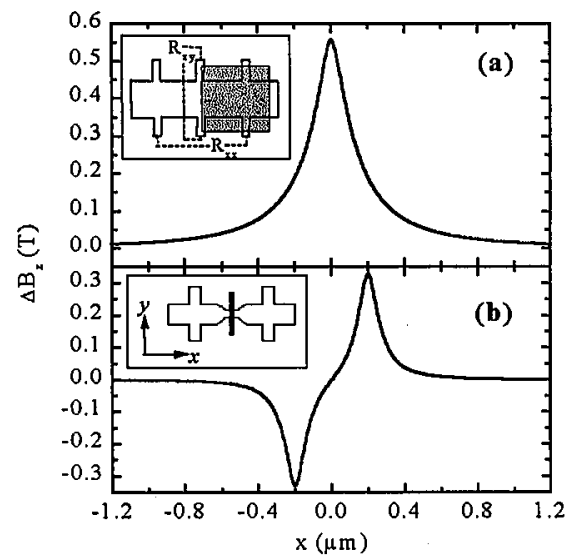

Fig. 1. (a) Simple magnetic barrier. Calculated magnetic field profile at the 2DEG for saturation $M_{x}$. Insert: schematic of the device [19]. (b) Magnetic barrier due to a ferromagnetic stripe. Calculated magnetic field profile at the 2DEG for saturation $M_{x}$. Insert: schematic of the device [16].

sweeping an external magnetic field applied in the $x$ direction, it is possible to change $M_{x}$ continuously between positive and negative saturation magnetization, and thereby vary the amplitude and the sign of the inhomogeneous field profile that the 2DEG experiences.

Figures 2 and 3 show the measured $R_{x x}$ and $R_{x y}$ at $T=1.3 \mathrm{~K}$ for a full sweep through the hysteresis loop of the magnetic element. As $B_{\text {ext }}$ is swept from $-0.4 \mathrm{~T}$ to $+0.4 \mathrm{~T}, R_{x x}$ decreases from its maximum value (for $M_{x}=-M_{\text {sat }}$ ) to a minimum at the coercive field of the Co element $\left(M_{x}=0\right)$, rising back to the maximum value when $M_{x}$ reaches $+M_{\text {sat }}$. For the same $B_{\text {ext }}$ sweep $R_{x y}$ changes from a negative to a positive value. The use of $R_{x y}$ in this geometry as a possible magnetic field sensor has already been considered in some detail $[19,22,23,25,26]$. We find that the measured $R_{x y}$ at saturation, is within $5 \%$ of that calculated using the average magnetic field in the Hall cross [19].

The width of the magnetic barrier in the current direction is very much smaller than the electron mean free path and so the resistance due to the bar-

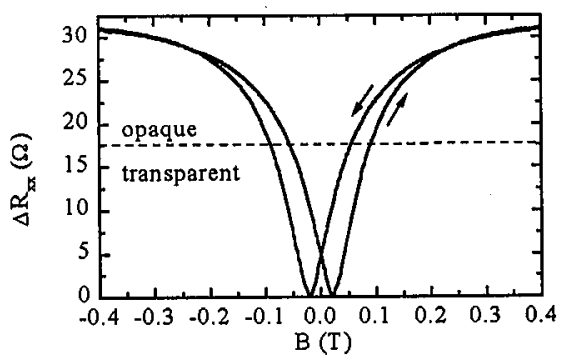

Fig. 2. Measured $\Delta R_{x x}$ as a function of external in-plane magnetic field. 


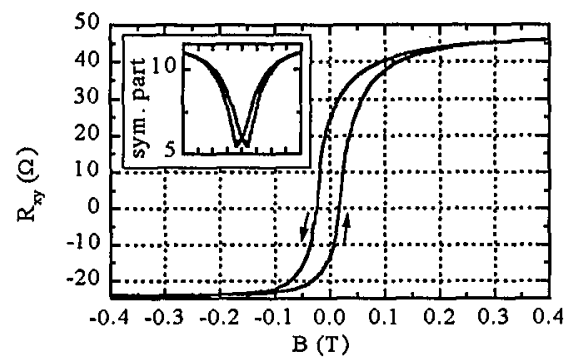

Fig. 3. Measured $R_{x y}$ as a function of external in-plane magnetic field. The insert shows the symmetric part of the measured $R_{x y}$.

rier will be dependent on the ballistic transmission probability. This probability depends only upon the initial angle between the $y$-axis and the trajectory of an electron, entering the barrier region from the left-hand side. Above a critical angle, the electron is transmitted; below this angle, the electron is reflected due to the action of the Lorentz force. This is illustrated in Fig. 4. The critical angle, for an electron with velocity $v$, is given by

$$
\phi_{\mathrm{c}}=\arccos (1-e \Phi / m v) \text { with } \Phi=\int B_{z}(x) \mathrm{d} x .
$$

$\Phi$ is the integrated $z$-component of the magnetic flux of the barrier. In our geometry the functional form $B_{z}(x)$ is particularly simple and the integral can be solved analytically to give $\phi_{\mathrm{c}}=d \mu_{0} M_{x} / 2$, where $M_{x}$ is the $x$ component of the magnetisation of the ferromagnetic element and $d$ is its thickness. If $\phi_{\mathrm{c}}<\pi$, we are in the "transparent" regime and some ballistic transmission is allowed (see Fig. 4). The conductance of the barrier decreases with increasing barrier height as fewer and fewer electrons are transmitted. For $\phi_{\mathrm{c}}>\pi$, all electrons are reflected and the semiclassical ballistic conductance equals zero, this we refer to as the "opaque" regime.

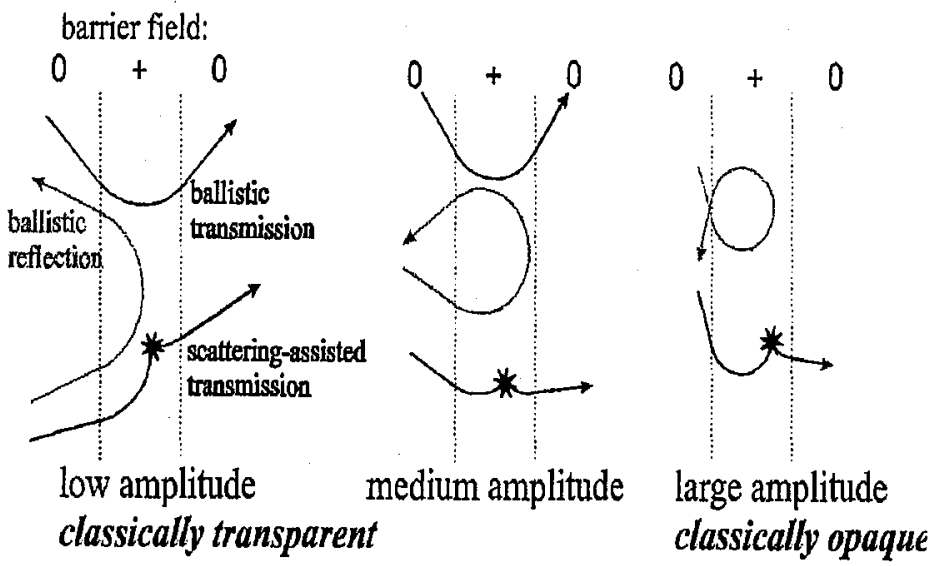

Fig. 4. Illustration of the types of semiclassical trajectories for a simple magnetic barrier. 
Since we have found that $R_{x y}$ is proportional to $\Phi$ we can obtain the functional form of $R_{x x}(\Phi)$ experimentally by plotting $R_{x x}$ as a function of $R_{x y}[17,19]$. Figure 2 shows that with increasing external field we are moving continuously from the "transparent" to the "opaque" in our samples. The measured conductance, however, remains finite. This could be due to scattering-aided transmission or transmission via edge channels.

In the standard semiclassical approach [27] $\Delta R_{x x}$ can be calculated directly from the appropriate average ballistic transmission probability, which is given by

$$
\langle T\rangle=\left(1-\pi e d \mu_{0} M_{x} / h k_{\mathrm{F}}\right)
$$

and

$$
\Delta R_{x x}=\left(h / 2 e^{2}\right)\left(\pi / w k_{\mathrm{F}}\right)(1-\langle T\rangle) /\langle T\rangle .
$$

This expression gives infinite $\Delta R_{x x}$ in the opaque regime; it also predicts that $R_{x x}$ should increase linearly with $M_{x}$ and thus with $R_{x y}$ for small barrier heights. However, experimentally we observe an approximately quadratic dependence.

Figure 5 shows clear evidence for a qualitative difference in the behavior of $R_{x x}$ between the transparent and opaque regime. In the transparent regime, the additional resistance due to the presence of the magnetic barrier, $\Delta R_{x x}=$ $R_{x x}\left(B_{\text {ext }}\right)-R_{x x}\left(B_{\text {ext }}=0\right)$, is temperature independent up to $\approx 40 \mathrm{~K}$ although the total resistance, and thus the scattering rate, has increased by a factor of $\approx 2$. This behavior is consistent with ballistic transmission across the barrier. In the opaque regime, $\Delta R_{x x}$ is found to decrease with increasing temperature over the whole temperature range. For the case of Fig. 5 the negative temperature dependence of $\Delta R_{x x}$ is so large that the total resistance measured in the opaque regime has a negative temperature coefficient of resistance. This is what one would expect if the transmission were accomplished by scattering within the barrier region. The resistance at zero barrier magnetization, $R_{0}$, should be simply proportional to the scattering rate. Figure 6 shows that the barrier conductance, $G=1 / \Delta R_{x x}$, increases linearly with $R_{0}$ and therefore with the scattering rate confirming the role of scattering assisted conductance. However, the intercept at zero $R_{0}$ indicates that the conductance would still be far from zero in the absence of scattering. The probable reason for this is that edge state transport is playing a significant role. For

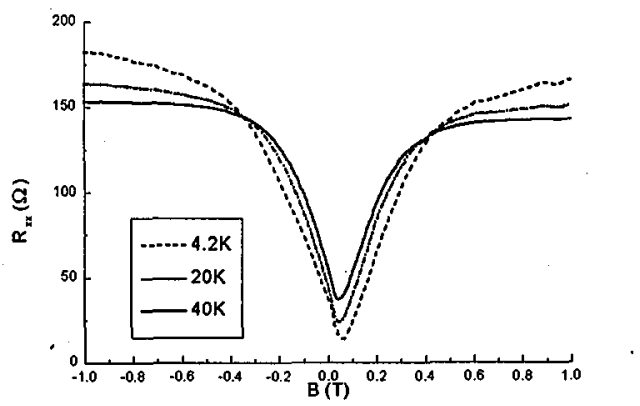

Fig. 5. Temperature dependence of $R_{x x}$ due to a large amplitude simple magnetic barrier. 


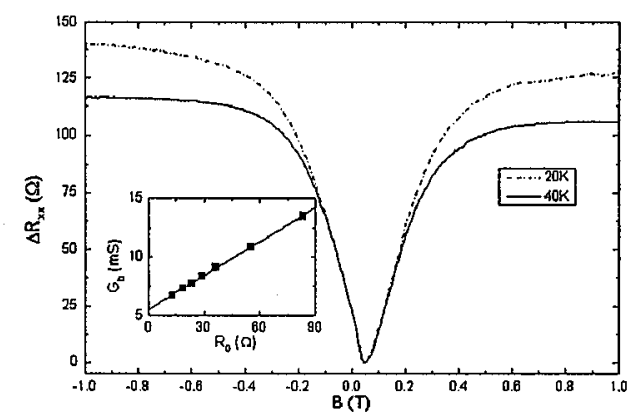

Fig. 6. Temperature dependence of $\Delta R_{x x}$. Insert: dependenceof the barrier conductance on $R_{0}$.

$\phi_{\mathrm{c}}>\pi$ the magnetic barrier has stationary bound states and corresponding edge states which can transmit electrons through the barrier. These states correspond to semiclassical skipping orbits at the physical edges of the conducting channel. When we include the contribution to the conductance of these states, the divergence of the resistance for $\phi_{\mathrm{c}}>\pi$ is removed and much better agreement with the experimental results is obtained.

In summary, we find that large-amplitude magnetic barrier across a hybrid Hall bar device can produce very large changes in $R_{x x}$. At low barrier heights the measured temperature dependence of $\Delta R_{x x}$ is consistent with ballistic conductance but the functional form of $\Delta R_{x x}\left(M_{x}\right)$ is not. In the classically opaque regime transmission through the barrier is scattering assisted or via edge states. Despite these conduction mechanisms we obtain magnetoresistances of $\approx 1000 \%$ at $4.2 \mathrm{~K}$, falling to $\approx 1 \%$ at room temperature, a value that is of technological importance.

\section{Submicron magnetic barriers due to single magnetic stripes: nanomagnetometry}

We have also studied the resistance changes produced by more complex magnetic barriers $[15,16]$ that are produced at a 2DEG by single submicron cross-section ferromagnetic stripes places across constricted Hall bars (see Fig. 1b). These devices show distinctly different behavior to those with simple barriers. Such devices can also serve as nanomagnetometers.

The polycrystalline cobalt stripes studied are tens of microns long (in the $y$-direction), 300 to $400 \mathrm{~nm}$ wide and 60 to $120 \mathrm{~nm}$ high. The center of the stripe is considered to be at $x=0$. For $B_{\text {ext }}=0$, the magnetization $M$ lies along the long axis of the line due to shape anisotropy. In this case, the $z$-component of the stray field at the $2 \mathrm{DEG}, B_{z}$, is negligibly small in the active region of the device. When $B_{\text {ext }}$ is applied in the $x$-direction, $M$ rotates towards this direction, inducing a positive $B_{z}$ underneath one edge and a negative $B_{z}$ underneath the other edge. The amplitude of $B_{z}$ is proportional to the $x$-component of $M$ and is shown in Fig. 1b for the case of saturation ( $B_{\text {ext }}$ large enough to force $M$ along $x$ ) for a $350 \mathrm{~nm}$ wide $120 \mathrm{~nm}$ thick cobalt line and with a 2DEG $35 \mathrm{~nm}$ below the surface. This field profile is independent of $y$ in the active region of the Hall bar and it is 
antisymmetric in $x$ about the line's center, so the average $B_{z}$ is zero. The spatial extent of the region where $\left|B_{z}(x)\right|$ is significantly larger than zero is much smaller than the electron mean free path. The field profile is just the superposition of a positive and a negative peak each with the same functional form as that for the simple barrier shown in Fig. 1a.

For this type of magnetic barrier, three different types of semiclassical electron trajectories exist. An electron impinging onto the barrier at angle $\phi_{\mathrm{i}}$ to the $y$-axis larger than a critical angle $\phi_{\mathrm{c}}$ is transmitted through the barrier without a change of direction. For $\phi_{\mathrm{i}}$ less than $\phi_{\mathrm{c}}, \phi(x)$ goes through zero before $x=0$ is reached and the electron is reflected. The critical angle is defined by the trajectory for which $\phi(0)=0$, and it is given by Eq. (1) with the integrated flux being evaluated for $-\infty<x<0$. There also exist trapped orbits inside the barrier; so-called "snake orbits". An electron crossing the center line at an angle $\pi-\phi_{\mathrm{c}}<\phi(0)<\pi+\phi_{\mathrm{c}}$ is effectively reflected back towards the center line and is channeled along this line in the $y$-direction.

Figure 7a shows $R_{x x}$ as a function of $B_{\text {ext }}$. As $B_{\text {ext }}$ is swept from $-0.6 \mathrm{~T}$ to +0.6 T, $M_{x}$ varies from its negative saturation value through zero to the positive saturation value. This changes the barrier amplitude and the critical angle, and hence the relative number of the different orbit types. Because only the first orbit type contributes directly to the conduction through the barrier, the longitudinal resistance $R_{x x}$ increases as $\left|M_{x}\right|$ increases. The barriers produced by the magnetic stripes studied so far are of smaller magnitude, at saturation, than those considered in Sec. 3 and are always in the "transparent" regime.
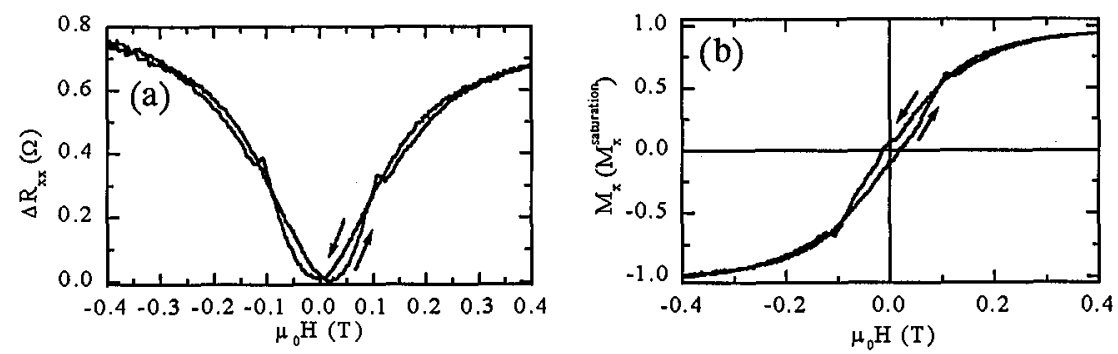

Fig. 7. (a) $\Delta R_{x x}$ as a function of $B_{\text {ext }}$ for $B_{\text {ext }}$ in the plane of the 2DEG at an angle of $9^{\circ}$ to the $x$-direction. (b) The normalized magnetization loop deduced from the resistance measurements of Fig. 7 a.

We find that $\Delta R_{x x}$ increases proportionally to the resistivity of the 2DEG with increasing $T$ up to about $40 \mathrm{~K}$. This contrasts with what we find for the simple magnetic barrier discussed in Sec. 3. For ballistic transmission and reflection, $\Delta R_{x x}$ should be independent of $T$. For scattering assisted transmission $\Delta R_{x x}$ would reduce with increasing $T$. We therefore conclude that scattering into and out of snake orbits is important for this barrier topology. We have developed a theory for the resistance change due to the presence of snake orbits [14] the predictions of which agree well with our experimental results. We have suggested that hybrid devices of this type can be used as nanomagnetometers with extremely high 
sensitivities and have shown that it is possible to obtain detailed quantitative information on hysteresis and switching in single submicron magnetic stripes $[16,17]$. This is illustrated in Fig. 7b which shows the magnetisation of a single submicron Co stripe obtained from the $\Delta R_{x x}$ data of Fig. 7a. The nature of the hysteresis loops obtained and the origin of the magnetisation switching events apparent in the data are discussed in detail in Refs. [15] and [16].

\section{Periodic magnetic fields}

The transport properties of two-dimensional electron systems in 1D and 2D periodically modulated magnetic fields have been studied extensively [7-14]. The type of device used is illustrated schematically in Fig. 8. We will present results for the case of an array of cobalt stripes, oriented normal to the [100], with period $a=500 \mathrm{~nm}$, width $d=200 \mathrm{~nm}$ and height $h=120 \mathrm{~nm}$. Figure 9 shows measured $R_{x x}$ plotted against $z$-component of the external magnetic field, $B_{\text {ext }, z}$, for $B_{\text {ext }}$ perpendicular to the plane of the 2DEG $\left(\phi=0^{\circ}\right.$ ) and at $\phi=80^{\circ}$ (note the logarithmic scale). The strong low field magnetoresistance oscillations are not Shubnikov-de Haas oscillations but result from commensurability between the diameter of the cyclotron orbit at the Fermi level and the period of the magnetic modulation [7-14]. These commensurability oscillations are closely related to those

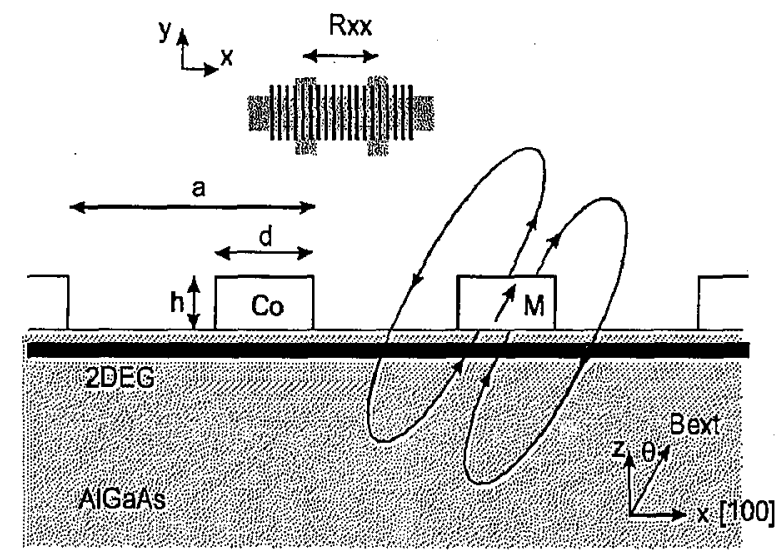

Fig. 8. Typical device structure in which a ferromagnetic grating produces a periodic magnetic field at the 2DEG.

seen for electrostatic modulation $[7,8]$. For $\phi=80^{\circ}$ an additional very large low field magnetoresistance is also present. This arises because, at large $\phi$, the amplitude of the $z$-component of the sign alternating magnetic field, due to the magnetisation of the stripes, is larger than $B_{\text {ext, } z}[12-14]$ as is shown in Fig. 10.

In the presence of a uniform magnetic field the semiclassical electron trajectories are circular. For a magnetic field which is periodic but which never changes sign the semiclassical electron trajectories are cyclotron-like. The rate of drift of these states in the $y$-direction varies periodically in $1 / B_{\mathrm{ext}, z}$, giving rise to the commensurability oscillations $[7,8]$. For a periodic sign alternating magnetic field there also exist open "snake" orbits centered on the lines of zero field. All electrons 


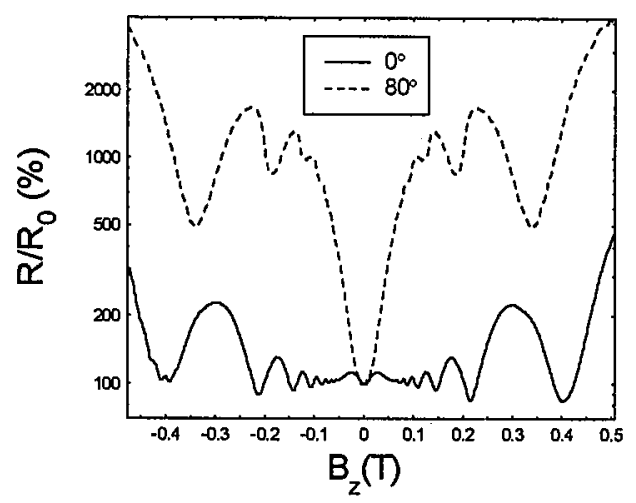

Fig. 9. $\quad R_{x x}$ measured at $1.3 \mathrm{~K}$ for the $B_{\text {ext }}$ perpendicular to the $2 \mathrm{DEG}\left(\phi=0^{\circ}\right)$ (solid line) and at $\phi=80^{\circ}$ (dashed line). $R_{0}$, the resistance at $B=0$, is $58 \Omega$.

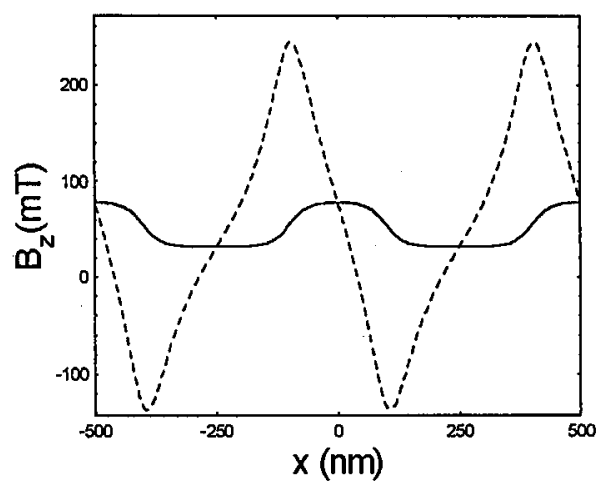

Fig. 10. The calculated $B_{z}$ for $B_{\text {ext }, z}=50 \mathrm{mT}$ for $\phi=80^{\circ}$ (solid line) and at $\phi=80^{\circ}$ (dashed line).

crossing a $B=0$ line at an angle $\phi$ smaller than the minimum angle $\phi_{\mathrm{c}}$ are trapped in these "snake" orbits. These "snake" orbits are the same as those for the single stripes considered in Sec. 4 and $\phi_{c}$ is exactly the same. These open "snake" orbits propagate with drift velocities close to the Fermi velocity, while the cyclotron-like orbits are almost closed. To a good approximation one can then write the expected magnetoresistance as [12]

$$
\Delta R_{x x} / R_{0}=\left(2 \phi_{\mathrm{c}} / \pi\right)\left(\mu B_{\mathrm{ext}, z}\right)^{2} .
$$

The magnetic field profile at the $2 \mathrm{D}$ electrons is relatively easy to calculate and, when this is used to calculate $\phi_{c}$ Eq. (3) gives good agreement with the measured low field magnetoresistance [12-14]. At low temperatures the temperature dependence of $\Delta R_{x x} / R_{0}$ is found to follow that of $\mu^{2}$ [14].

When $B_{\text {ext }}$ is applied in the $x$-direction its only effect is to rotate the magnetisation of the magnetic stripes into this direction and thus turn on the magnetic modulation. The modulation then has a mean value of zero. This leads to a 
$\Delta R_{x x} / R_{0}$ that, at low temperatures, should only depend on $\phi_{\mathrm{c}}$ and which should be temperature independent $[12,16]$. Remarkably $\Delta R_{x x}$ is found experimentally to increase as $\sim T^{2}$ at low temperatures [13]. This is very hard to understand on the basis of the otherwise very successful semiclassical model. We have suggested [13] that this is due to electron-electron scattering and this idea has been investigated further [11]. However, the basic mechanism by which electron-electron scattering is able to relax the total electron momentum is as yet unclear.

\section{Spatially random magnetic fields}

The transport properties of a two-dimensional electron gas (2DEG) in the presence of a spatially random magnetic field has attracted great theoretical interest recently $[3,28,29]$, largely due to its relevance for the study of composite fermions $[3,28]$. When the resistivity is dominated by the disordered electrostatic potential, the random magnetic field can be considered as a perturbation [29-31]. For large amplitude random magnetic fields with correlation lengths which are small compared to the electron mean free path (due to electrostatic disorder) the situation is much more interesting. The semiclassical trajectories are "snake orbits" which are guided along lines of zero magnetic field and drifting cyclotron-like or-

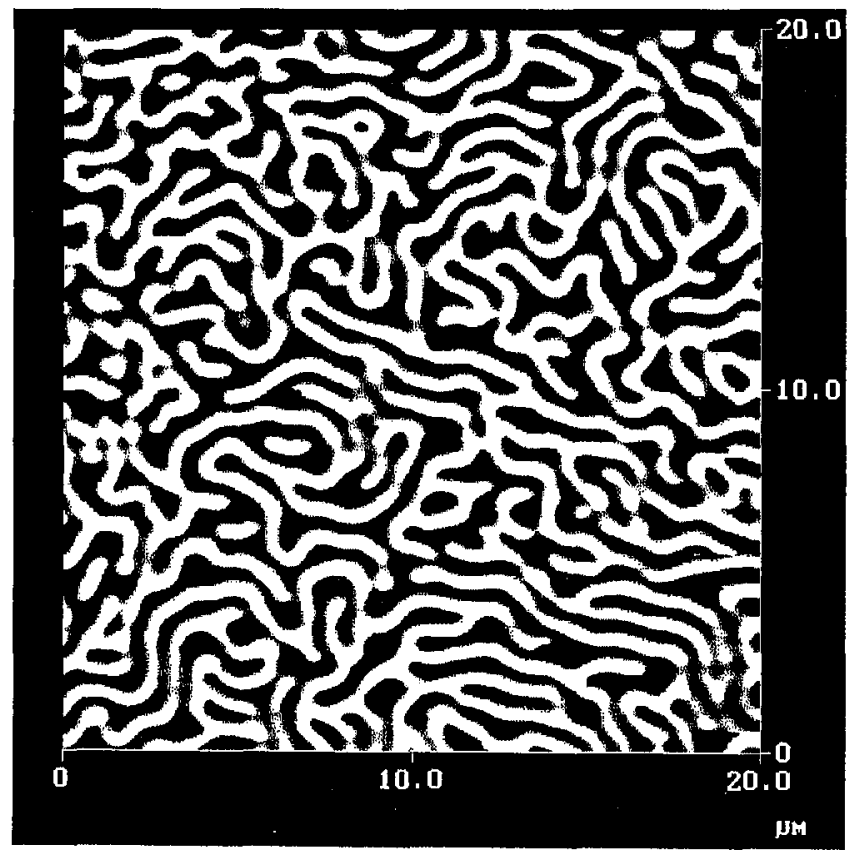

Fig. 11. Magnetic force microscope image of the CoPd multilayer films in the presence of an external magnetic field of $+0.3 \mathrm{~T}$ applied perpendicular to the plane of the multilayers. The light and dark regions represent domains pointing up and down (into and out of the page). Dark regions are in the direction of the external field. 
bits which are guided along contours of constant $B_{z}$ [3]. These are similar to the trajectories for the periodic case. The key difference is that these contours are generically closed and the conductance arises from scattering [3].

Experimental data exists for very weak random magnetic fields [29-31], and random magnetic fields with correlation lengths approximately equal [6] or much larger than [4] the electron mean free path. However, the theories of the effects of large amplitude random magnetic fields with correlation lengths which are small compared to the electron mean free path have, until now, not been tested due to the absence of appropriate experimental data. We here recently been able to study the effect of well characterized, large amplitude random magnetic field with a correlation length much smaller than the electron mean free path.

Our samples are created by depositing continuous CoPd multilayers films onto the surface of our high mobility, near surface 2DEG layers. The films completely cover the active region of the Hall bars. These multilayers have the property that, at certain points in the magnetisation loops, maze-like domain patterns form, magnetized perpendicular to the plane of the multilayers (see Fig. 11). The domains, which are of the order of $500 \mathrm{~nm}$ in size, produce a random magnetic field at the plane of the 2DEG. This has been confirmed by magnetic force microscope (MFM) and vibrating sample magnetometer (VSM) measurements. This property of the multilayers enables us to switch the random field on and off by applying an external magnetic field perpendicular to the plane of the multilayers. As Fig. 12 shows we see a large hysteretic magnetoresistance in the presence of the random field that shows many interesting features. As Fig. 11 shows we are able to obtain detailed information about the spatial variation of the random magnetic field from MFM images taken in the presence of an external magnetic field. We are able to use the MFM images, along with VSM and SQUID measurement, to obtain the spatially varying random magnetic field at the $2 \mathrm{DEG}$. The correlation function of the random magnetic field, which is a function of the external magnetic field,

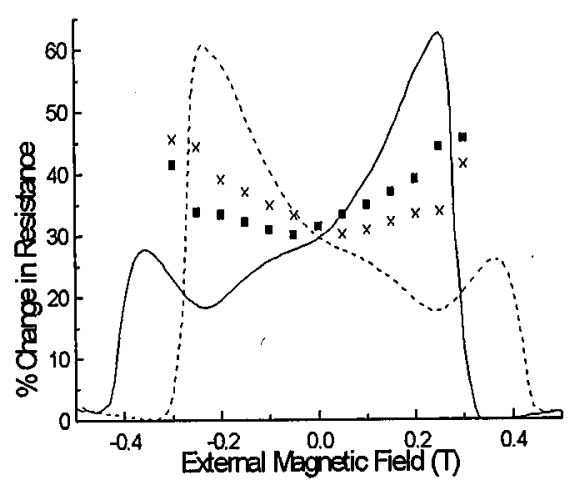

Fig. 12. The magnetoresistance measured at $4.2 \mathrm{~K}$. The solid line is for the field sweeping down and the dotted line is for the field sweeping up. The theoretical fit is represented by squares (field sweeping down) and crosses (field sweeping up). 


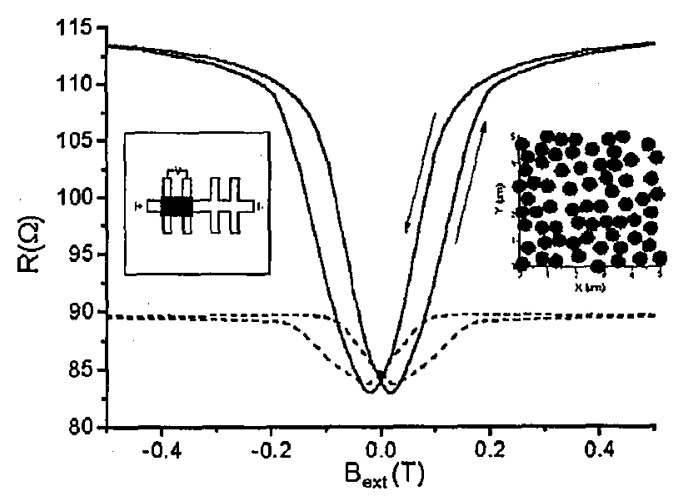

Fig. 13. $R_{x x}$ measured for a random magnetic field produced by randomly placed $300 \mathrm{~nm}$ diameter Co dots. The in-plane field produces an anisotropic magnetoresistance. The resistance is larger when the current direction and the in-plane field are parallel than when they are at $90^{\circ}$.

can then be used in the analytical theories. The results of one such comparison is shown in Fig. 12. We have also performed Monte Carlo calculations using the experimentally derived random magnetic field profiles. Our initial results show that reasonable agreement between experiment and the predictions of the analytical theory [29] can only be obtained if the relaxation time is taken to be close to the scattering time rather than the momentum relaxation time.

Very recently we have studied random magnetic fields produced by randomly positioned submicron magnetic dots (see Fig. 13). This system is particularly interesting because an in-plane external magnetic field can be used to introduce a controllable anisotropy into the random magnetic field. For the external magnetic field parallel to the current, the contours of zero $B_{z}=0$ are preferentially orientated perpendicular to the current direction. For the external magnetic field perpendicular to the current the contours of zero $B_{z}=0$ are preferentially orientated parallel to the current direction. This leads to an anisotropic $R_{x x}$ due to the "snake" states which are guided along these contours. This provides direct evidence of the role of these particular guided trajectories.

\section{Conclusions}

Hybrid semiconductor/ferromagnet devices show a wide range of interesting new transport properties. Many are semiclassical in origin and can be understood on the basis of the allowed semiclassical trajectories. Despite the seeming simplicity of electron transport through simple magnetic barriers we still do not have a quantitative theory capable of explaining all the experimental observations. Drifting cyclotron states and "snake" states have been shown to play key roles in the transport properties of more complex barriers, periodic magnetic fields and random magnetic fields. Hybrid semiconductor/ferromagnet devices show new types of giant magnetoresistance and large magnetoresistance at room temperature should be possible with device optimization. These devices have potential as magnetic 
field sensors, memory or logic elements, and nanomagnetometers. The semiclassical effects observed in non-uniform magnetic fields are relatively simple, very interesting and potentially useful.

\section{Acknowledgments}

We gratefully acknowledge financial support by the EPSRC (U.K.) and the ESPRIT MEL/ARI SPIDER program (EU).

\section{References}

[1] For a recent review see: F.M. Peeters, Jo De Boeck, in: Handbook of Nanostructured Materials and Nanotechnology, Ed. H.S. Nalwa, Vol. 3, Academic Press, New York 1999 , p. 345.

[2] S.D.M. Zwerschke, A. Manolescu, R.R. Gerhardts, Phys. Rev. B 60, 5536 (1999) and references therein.

[3] A.D. Mirlin, J. Wilke, F. Evers, D.G. Polyakov, P. Wolfle, Phys. Rev. Lett. 83, 2801 (1999); F. Evers, A.D. Mirlin, D.G. Polyakov, P. Wolfle, Phys. Rev. B, in press; L. Zielinski, K. Chaltikian, K. Birnbaum, C.M. Marcus, K. Campman, A.C. Gossard, Europhys. Lett. 42, 73 (1998) and references therein.

[4] F.B. Mancoff, R.M. Clarke, C.M. Marcus, S.C. Zhang, K. Campman, A.C. Gossard, Phys. Rev. B 51, 13269 (1995); F.B. Mancoff, L.J. Zielinski, C.M. Marcus, K. Campman, A.C. Gossard, Phys. Rev. B 53, R7599 (1996).

[5] M.L. Leadbeater, C.L. Foden, J.H. Burroughes, M. Pepper, T.M. Burke, L.L. Wang, M:P. Grimshaw, D.A. Ritchie, Phys. Rev. B 52, 8629 (1995).

[6] G.M. Gusev, U. Gennser, X. Kleber, D.K. Maude, J.C. Portal, D.I. Lubyshev, P. Basmaji, M. de PA Silva, J.C. Rossi, Yu.V. Nastaushev, Surf. Sci. 361-362, 855 (1996); G.M. Gusev, J.R. Leite, A.A. Bykow, N.T. Moshegov, V.M. Kudryashev, A.I. Toporov, Y.V. Nastaushev, Phys. Rev. B 59, 5711 (1999).

[7] H.A. Carmona, A.K. Geim, A. Nogaret, P.C. Main, T.J. Foster, M. Henini, S.P. Beaumont, M.G. Blamire, Phys. Rev. Lett. 74, 3009 (1995).

[8] P.D. Ye, D. Weiss, R.R. Gerhardts, M. Seeger, K. von Klitzing, K. Eberl, H. Nickel, Phys. Rev. Lett. 74, 3013 (1995); Surf. Sci. 361-362, 337 (1996).

[9] P.D. Ye, D. Weiss, R.R. Gerhardts, H. Nickel, J. Appl. Phys. 81, 5444 (1997).

[10] S. Izawa, S. Katsumoto, A. Endo, Y. Iye, J. Phys. Soc. Jap. 64, 706 (1995).

[11] M. Kato, A. Endo, Y. Iye, Phys. Rev. B 58, 4876 (1998); M. Kato, A. Endo, M. Sakairi, S. Katsumoto, Y. Iye, J. Phys. Soc. Jap. 68, 1492 (1999); M. Kato, A. Endo, S. Katsumoto, Y. Iye, J. Phys. Soc. Jap. 68, 2870 (1999).

[12] A. Nogaret, S. Carlton, B.L. Gallagher, P.C. Main, M. Henini, R. Wirtz, R. Newbury, M.A. Howson, S.P. Beaumont, Phys. Rev. B 55, 16037 (1997).

[13] N. Overend, A. Nogaret, B.L. Gallagher, P.C. Main, R. Wirtz, M. Henini, R. Newbury, M.A. Howson, S.P. Beaumont, Physica B 249/251, 326 (1998).

[14] N. Overend, A. Nogaret, B.L. Gallagher, P.C. Main, M. Henini, C.H. Marrows, M.A. Howson, S.P. Beaumont, Appl. Phys. Lett. 72, 1724 (1998).

[15] V. Kubrak, F. Rahman, N. Overend, B.L. Gallagher, P.C. Main, M. Henini, J. de Boeck, M. Behest, C.H. Marrows, M.A. Howson, Physica B 256/258, 380 (1998).

[16] V. Kubrak, F. Rahman, B.L. Gallagher, P.C. Main, M. Henini, C.H. Marrows, M.A. Howson, Appl. Phys. Lett. 74, 2507 (1999). 
[17] V. Kubrak, A.C. Neumann, B.L. Gallagher, P.C. Main, M. Henini, C.H. Marrows, M.A. Howson, Physica E 6, 755 (2000).

[18] A.W. Rushforth, B.L. Gallagher, P.C. Main, A.C. Neumann, C.H. Marrows, I. Zoller, M.A. Howson, B.J. Hickey, M. Henini, Physica E 6, 751 (2000).

[19] V. Kubrak, A.C. Neumann, B.L. Gallagher, P.C. Main, M. Henini, C.H. Marrows, B.J. Hickey, J. Appl. Phys. 87, 5986 (2000).

[20] V. Kubrak, A.W. Rushforth, A.C. Neumann, F. Rahman, B.L. Gallagher, P.C. Main, M. Henini, C.H. Marrows, B.J. Hickey, Physica E 7, 997 (2000).

[21] K. Geim, S. v. Dubonos, J.G.S. Lok, I.V. Grigorieva, J.C. Maan, L. Theil Hansen, P.E. Lindelof, Appl. Phys. Lett. 71, 2379 (1997).

[22] M. Johnson, B.R. Bennett, M.J. Yiang, M.M. Miller, B.V. Shanabrook, Appl. Phys. Lett. 71, 974 (1997).

[23] F.G. Monozon, M. Johnson, M.L. Roukes, Appl. Phys. Lett. 71, 3087 (1997).

[24] H. Davies, I.A. Larkin, Phys. Rev. B 49, 4800 (1994); E. Skuras, A.R. Long, I.A.C. Holland, Appl. Phys. Lett. 70, 871 (1997).

[25] S. Ibrahim, A. Schweigert, F.M. Peeters, Phys. Rev. B 56, 7508 (1997).

[26] F.M. Peeters, X.Q. Li, Appl. Phys. Lett. 72, 572 (1998).

[27] C.W.J. Beenakker, H. van Houten, Phys. Rev. Lett. 63, 1857 (1989).

[28] A.D. Mirlin, D.G. Polyakov, P. Wölfe, Phys. Rev. Lett. 80, 2429 (1998).

[29] P. Hedegard, A. Smith, Phys. Rev. B 50, 14726 (1994); Phys. Rev. B 51, 10896 (1995).

[30] P.D. Ye, D. Weiss, G. Lütjering, R.R. Gerhardts, K. von Klitzing, K. Eberl, H. Nickel, G. Weimann, in: Proc. 23rd Int. Conf. on Physics of Semiconductors, Eds. M. Scheffler, R. Zimmermann, World Sci., Singapore 1996, p. 1529.

[31] A.K. Geim, S.J. Bending, I.V. Grigorieva, Phys. Rev. Lett. 69, 2252 (1992). 